Man and Nature

L'homme et la nature

\title{
La femme virile - la femme-héros dans le théâtre de la Terreur
}

\section{Annette Graczyk}

Volume 11, 1992

URI : https://id.erudit.org/iderudit/1012669ar

DOI : https://doi.org/10.7202/1012669ar

Aller au sommaire du numéro

Éditeur(s)

Canadian Society for Eighteenth-Century Studies / Société canadienne d'étude du dix-huitième siècle

ISSN

0824-3298 (imprimé)

1927-8810 (numérique)

Découvrir la revue

Citer cet article

Graczyk, A. (1992). La femme virile - la femme-héros dans le théâtre de la Terreur. Man and Nature / L'homme et la nature, 11, 25-34.

https://doi.org/10.7202/1012669ar

Copyright (C Canadian Society for Eighteenth-Century Studies / Sociéte canadienne d'étude du dix-huitième siècle, 1992
Ce document est protégé par la loi sur le droit d'auteur. L'utilisation des services d'Érudit (y compris la reproduction) est assujettie à sa politique d'utilisation que vous pouvez consulter en ligne.

https://apropos.erudit.org/fr/usagers/politique-dutilisation/ 


\section{La femme virile - la femme-héros dans le théâtre de la Terreur}

Sous l'influence directe de la Révolution, le théâtre change foncièrement de caractère. Devenu dans une large mesure théâtre d'actualité et occupant un rôle prédominant dans la culture révolutionnaire quotidienne, il devient un nouveau média de masses soumis à une utilisation clairement pédagogique. ${ }^{2}$ Les Jacobins y recourent pour accélérer cette transformation républicaine et égalitaire de la société qui constitue leur programme politique. Le 2 août 1793 est décrétée la politisation du spectacle: on se propose de privilégier et de représenter, en partie gratuitement, les pièces modèles exaltant les vertus patriotiques ou mettant en scène les événements glorieux de la toute récente histoire révolutionnaire; l'incivisme sur scène devient passible d'arrestation. Cette loi du 2 août va à la fois amorcer une vague de purges dans les salles et transformer le théâtre dans une large mesure en école politicomorale, en temple d'exaltation républicaine. Sur le plan esthétique, cette mise en scène exemplaire de la Révolution conduit à une stylisation symbolique des événements et de l'action, et à un foisonnement des héros-citoyens dont je me propose d'analyser les prototypes féminins.

Dans les toutes premières pièces, la lutte pour la liberté va de pair avec des idées anarchisantes qui associent à l'idée de liberté celle de la libération érotique. Mais ces tendances hédonistes ou tendances à la transgression des normes, cèdent bien vite le pas à un théâtre placé sous l'égide de la vertu. Pendant la domination jacobine, la féminité devient sur scène accessoire d'un paysage bucolique. Elle symbolise l'état d'innocence naturelle que la Révolution veut réinstaurer dans toute son évidence première. C'est au fond l'assimilation de la femme à la nature, telle que la pratique la philosophie de la Nature des Lumières, qui fait d'elle cet excellent élément de la symbolique révolutionnaire et lui confère ses propriétés allégoriques de déesse de la liberté. Ce retour à l'Age d'or s'inscrit en réaction contre le mode de vie dénaturé de l'aristocratie féodale.

A l'instar du personnage du Bon sauvage qui n'est pas moins fictif, la «nature» féminine prend les traits d'une nature domestiquée dont les 
aspects menaçants sont jugulés par l'assignation à son rôle social d'épouse et de mère.

Quand on attend des femmes engagement civique et patriotisme, on ne les sollicite que dans le cadre restreint de leur féminité: la femme incarne et contrôle l'aspect émotionnel de la Révolution. Elle sert d'instance régulatoire des émotions tout en influençant le civisme et le patriotisme de son mari et de ses enfants. La justesse de son «instinct» social et politique exclut les mésalliances et fait qu'elle démasque toujours les contre-révolutionnaires et les opportunistes.

Il y a pourtant, même au théâtre, des situations dans lesquelles les femmes vont au delà des normes de la féminité établies par la philosophie de la Nature des Lumières et dans lesquelles certaines protagonistes se surpassent en faisant preuve d'un courage rare. On peut distinguer les trois types suivants de citoyennes-héros, que je vais analyser successivement dans leur contexte social et politique: le modèle de Judith, l'amazone et la mère spartiate. ${ }^{3}$ Elles sont autant d'exemples d'une virilisation de la femme et répondent au besoin de l'époque d'une auto-héroïsation décalquée sur le modèle de l'Antiquité, même là où elles sont utilisées à des fins de politique différente.

\section{JUDITH ET SES SOEURS}

Dans la tragédie de Gabriel Légouvé Epicharis et Néron, ou Conspiration pour la Liberté, ${ }^{4}$ une femme, Epicharis, se dresse avec courage contre l'usurpateur Néron qui règne sur la Rome républicaine en véritable despote. La pièce, qui plonge les spectateurs dans la Rome antique, sort le 3 février 1794, à une époque où la Terreur est à l'ordre du jour. Les Girondins ont péri sous la guillotine l'année précédente, et ce sera bientôt au tour de la nouvelle opposition, les Hébertistes et les Dantonistes, d'être mise en accusation. C'est un événement politique perturbateur qui motive la pièce de Légouvé ainsi que le personnage féminin: l'assassinat de Marat par Charlotte Corday le 13 juillet 1793.

Dans l'attentat de Charlotte Corday, les contemporains ont vu un renversement inquiétant de l'ordre des sexes que les anti-jacobins ont même considéré comme symptôme d'une crise politico-morale: les hommes sont relégués au rang d'eunuques, si bien qu'une femme doit assumer le rôle masculin pour remettre le monde d'aplomb.

Ainsi commence la pièce de Légouvé: Rome est tombée dans la débauche; la décadence et le vice règnent sur la vie publique, tandis que la vertu et l'amour de la liberté sont opprimés. La ville subit son esclavage en silence et n'oppose aucune résistance. Epicharis projette tout d'abord une action solitaire, un attentat contre Néron pour libérer 
Rome de son tyran. L'initiative politique que prend la femme est symptomatique d'une crise profonde de la morale républico-démocratique. C'est un appel à la virilité, à ces hommes trop peu mâles pour tenir tête à la fureur destructrice de Néron le tyran. Et le poète Lucain s'étonnant que ce soit une femme, Epicharis, qui mène le soulèvement, s'écrie:

\author{
O Lucain! quel affront! \\ Des coeurs républicains tu crus sentir la flamme, \\ Et tu n'as pas osé ce que tente une femme! \\ Pour t'affranchir d'un joug qui t'a trop abattu, \\ Une femme a besoin d'avertir ta vertu! (p. 17)
}

Mais Epicharis ne fait que déclencher le processus, la révolte même sera conduite par un homme, le consul Pison. Et ce dernier la détrompe: les actes $d$ 'héroïsme individuel ne peuvent pas venir à bout d'un despotisme établi, seul un vaste mouvement des masses le pourrait. Il est intéressant de noter qu'Epicharis saura mourir "virilement» sous les supplices de Néron. L'élévation héroïque requiert la suppression de la "nature» féminine. "La femme a disparu pour n'offrir qu'un héros», proclame la tragédie de Légouvé, qui passe pour une des réussites esthétiques de la tragédie révolutionnaire. Féminité et masculinité sont politiquement codées: Epicharis incarne le courage et l'énergie, Pison, triomphateur de Néron, le discernement politique. Néron, incapable de mourir est comme les hommes de son époque qui n'ont pas su l'entraver: efféminé.

Ce n'est qu'après la chute de Robespierre que la "nouvelle Judith", Charlotte Corday, deviendra un personnage de scène. La "tragédie» anonyme, parue en 1795 et intitulée Charlotte Corday, est une apothéose anti-jacobine de l'héroïne célèbre. La pièce replace les moments abstraits de Légouvé dans leur actualité politique, va même plus loin en avançant le jour de l'attentat au 31 mai, première journée insurrectionnelle contre les Girondins. Ici aussi, Charlotte Corday agit dans un moment où dans l'optique de l'opposition - l'action politique est paralysée, les députés girondins étant immobilisés par la violence populaire. Le fait que Charlotte a perpétré vraiment son attentat, intensifie davantage que dans la pièce de Légouvé l'idée de l'inversement des sexes. Charlotte Corday s'arroge un droit masculin, le droit de tuer. C'est pourquoi le thème politique du tyrannicide risque de se transformer en thème érotique. Jusqu'à nos jours, l'attentat historique de Charlotte Corday, qui n'a cessé d'inspirer les artistes, est chargé de symbolique castratrice, renforcée par le côté équivoque du meurtre dans la baignoire. La tragédie anonyme de 1795 tente de parer à cette érotisation en libérant 
la protagoniste de toutes connotations douteuses. Ainsi elle ne poignarde pas Marat dans la baignoire, mais à la Convention, dans un lieu politique et public. Elle-même a les traits d'une vestale, animée du feu pur de l'amour de la patrie et de la liberté. Sa chasteté virginale est fortement exaltée. Charlotte se comporte en virago, en vierge masculine. Elle ne devient héroïne qu'aux dépens de sa sexualité.

\section{L'AMAZONE}

Comme la virago, l'amazone représente un type de femme patriotique qui renverse à nouveau l'ordre des sexes, car elle pénètre dans le domaine soldatesque où l'action de tuer est réservée à l'homme. Alors que la virago est asexuée, l'amazone, elle, est une synthèse de féminité et d'héroïsme. Elle apparaît seulement dans une situation extrême, celle de la guerre. Son arrivée exprime une volonté de défense nationale, puisque l'effervescence du patriotisme atteint aussi un sexe qui n'était pas prédestiné au métier de soldat. Cela fait d'elle un aiguillon de la morale patriotique. Mais en même temps, elle véhicule un message foncièrement féministe, car, sur le terrain de Mars, elle prétend à l'égalité des sexes. Le beau sexe, oppose Pauline à son compagnon-patriote, dans une pièce de Philipon de la Madelaine,

[...] est aussi capable de grandes actions que le vôtre et celles qui font les héros, savent l'être. ${ }^{6}$

Le théâtre révolutionnaire reflète la fascination mais aussi l'irritation qu'exerce la femme-soldat sur les hommes, car s'ils en saluent l'expression d'une volonté de défense nationale, ils n'acceptent pas qu'elles menacent de leur échapper en s'engageant comme volontaires. Le soldat préfère garder sa Pénélope au foyer et le père, évoquant l'intérêt de $\mathrm{l}^{\prime}$ Etat, préfère penser à la perpétuation biologique de la République. L'amazone représente une menace potentielle, puisqu'elle fait éclater la cellule familiale prônée par la République jacobine.

Dans ces pièces on voit à quel point les revendications égalitaires des femmes mettent en cause le privilège masculin de porter des armes. La Révolution avait ôté à la Noblesse ce privilège et associé le droit à la citoyenneté à celui du service militaire. En armant le peuple exclu de la catégorie des citoyens, les Sans-culottes avaient exigé la participation des masses au pouvoir politique. L'amazone, en portant les armes, veut montrer qu'elle aussi aspire à la citoyenneté. Et c'est à travers la démon- 
stration d'égalité militaire que l'émancipation de la femme trouve sa vraie légitimation.

Ceci apparaît très clairement dans la pièce d'une féministe de renom: L'Entrée de Dumourier (!) à Bruxelles ou Les Vivandiers d'Olympes de Gouges. ${ }^{8}$ Dans la pièce surgissent les soeurs Ferning, les femmes-soldats les plus célèbres de l'époque, qui combattirent vraiment dans l'armée du Nord du général Dumouriez. «[...] la valeur n'a point d'âge ni de sexe,» clament les deux guerrières dans la pièce. «Imitez-nous", lance l'une des soeurs aux citoyennes de Bruxelles,

faisons plus aujourd'hui que les hommes; combattons pour défendre leurs droits, et vengeons en même temps notre sexe d'un tyrannique préjugé. Forçons la fierté, l'orgueil de ces superbes à rendre hommage à notre valeur; et qu'ils apprennent enfin que les femmes peuvent mourir à leurs côtés pour la cause commune de la patrie, et la destruction des tyrans.

En ce début 1793, Olympes de Gouges exprime encore l'espoir que la nouvelle constitution républicaine exaucera enfin l'exigeance d'une égalité universelle et qu'elle octroiera aux femmes ces droits qui leur avaient été refusé dans la première constitution de 1791. Dans la pièce, Dumourier déclare que les hommes de la République française se montreront équitables envers le sexe féminin, puisqu'il est indispensable que cette révolution naturelle s'étende sur tous indistinctement. Mais la pièce de la féministe De Gouges, qui en 1791 avait déjà revendiqué les droits de la femme et de la citoyenne, cette pièce échouera en 1793 auprès du public et sera suspendue dès la deuxième représentation.

\section{LA MÈRE SPARTIATE.}

A la différence de la virago et de l'amazone, chargées d'une inquiétante symbolique castratrice, la mère spartiate donne un modèle de «virilisation" sans le renversement des sexes ni la volonté de tuer. Bien au contraire, le personnage de la mère spartiate est une élévation au rang de Surmoi féminin.

La mère spartiate n'est mère que si l'héroïsme le permet. L'amour maternel, c'est-à-dire l'émotion qui justifie l'identité d'une mère, va être fonctionnalisé au profit des vertus patriotiques. Dans le conflit entre l'amour maternel émotionnel et les vertus républicaines, c'est l'amour égoïste pour l'enfant qui doit céder la place. Et même si les exigences républicaines triomphent des sentiments égoïstes de la mère, on assiste 
à une émotionnalisation des vertus républicaines, puisque celles-ci reçoivent de la mère la primauté: la mère spartiate est fière de son fils guerrier, même s'il perd la vie au combat.

L'héroïne réelle du théâtre républicain - c'est donc la mère, elle devient le principal véhicule du républicanisme. Son modèle antique est Cornélie, la célèbre mère des Gracques. Dans la tragédie de MarieJoseph Chénier de 1792, Caïus Gracchus, ${ }^{9}$ considérée par les Jacobins comme un véritable exemple de théâtre patriotique, c'est Cornélie qui défend les devoirs politico-moraux de son fils contre les exigences privées de son épouse Licinia:

Non, non, Licinia, n'abusez point son coeur;

Parlez de son devoir et non de son bonheur. (p. 8)

Lutte inutile puisque Gracchus est décidé à prendre la tête de son armée plébéienne. Face à l'inflexible républicaine Cornélie, la tentative de Licinia de sauver son mari est pure faiblesse féminine: Licinia clame dans un geste d'humilité au Consul Opimius:

Consul, n'écoute pas ses cris, son désespoir;

Au nom de son épouse, écoute la nature.

Et elle se jette à ses pieds, sous les yeux du parti du peuple horrifié.

Ah! je n'en rougis point, je suis épouse et mère. (pp. 24-25)

Les exigences de la société en guerre, les besoins de la Terreur favorisent de telles images, dans lesquelles l'instinct - ici l'instinct maternel - est sublimé en discernement politique. C'est pourquoi le prototype de la mère spartiate apparaît dans le théâtre de la Terreur, et tout particulièrement dans l'épisode légendaire de Barra, l'enfant-martyr de la guerre, sujet qui sera repris, parfois sous d'autres noms, dans une grande variété de pièces de l'An II. Apparaît ici le prototype d'un héroïsme double, celui du fils et de la mère, tel qu'on le voit déjà dans le titre: Barra ou La Mère républicaine. C'est la pièce d'une femme, Nicole Mathieu Villiers, ${ }^{10}$ qui entreprend, d'un point de vue féminin rarement exprimé, de faire là une justification du régime de terreur et de guerre. La féminité ne doit point dégénérer en faiblesse - c'est le reproche de l'énergique Dorothée à la sensible et conciliante Brigitte. Et elle va satisfaire à cette exigence en endossant le rôle de la mère spartiate. Dans la pièce de Madame Villiers, la mère est pour le jeune soldat une figure d'identification sacrée à la sur-mère Patrie et elle canalise l'amour de 
l'enfant en aptitude au sacrifice patriotique. Elle est aussi investie d'une autre mission politico-sociale: capable de sublimer la douleur de la perte de son fils en fierté républicaine, elle livre un modèle de travail de deuil collectif. Dans cette situation de déstabilisation interne, dans ce contexte de la perte d'êtres chers par la guerre ou la Terreur, on produit des modèles intégratifs de comportement civique qui induisent de surcroît un processus de compensation. Il faut donc des figures maternelles ad hoc. Quand le sacrifice est à l'ordre du jour, la promesse érotique d'une déesse de la liberté n'est plus d'aucune utilité. La sur-mère-Patrie est transcendée religieusement. La dernière caractéristique de la mère spartiate est qu'elle est le modèle d'un sacrifice cultuel: elle se sacrifie avec ses fils sur l'autel de la Patrie. Malgré la douleur qu'elle ressent à la mort de son fils, Dorothée, dans la pièce citée de Mme Villiers, n'en destine pas moins ses deux autres fils à l'armée:

La patrie n'a-t-elle pas sur eux des droits plus sacrés que les miens? [...] mes enfans, [...] moi, nous sommes tous à elle; nos veilles, nos sueurs, notre sang; tout lui appartient. [...]. Oh patrie! malheur a l'égoïste, dont l'insensible coeur n'est point embrasé de ton amour! mes enfans l'ont puisé dans mon sein; ils seront dignent de toi. [...]; je te les dévoue. (p. 55)

\section{LA VIRILISATION DE L'HOMME ET DE LA SOCIÉTÉ}

L'héroïsme exige donc de triompher de la nature féminine. Mais loin de n'affecter que les seules hérö̈nes, cette virilisation vaut aussi pour le héros masculin. Le modèle de ces personnages sacrifiant les exigences de la sphère privée à celles de la chose publique est puisé dans le réservoir des héros de l'Antiquité surtout romaine. L'Antiquité fournit le grand modèle sur lequel put s'appuyer déjà bien avant la Révolution - dans les domaines de l'esthétique et de la théorie -une morale politique d'action civique, structurée selon des critères rationaux. Avec la tragédie antique de l'époque révolutionnaire, la scène se transforme en tribune politique, et c'est dans le décor du forum romain qu'on discute des objectifs et de la conduite à tenir. L'exemple de Rome permet de s'exercer dans le jeu et la rhétorique du monde politique, et favorise en même temps ce don de soi à la chose publique qu'illustrent si bien les héros romains. Prendre modèle sur l'Antiquité signifie refouler tout ce qui appartient à la sphère privée et s'interdire de battre en retraite. Dans la tragédie de Chénier, Gracchus refoule à travers la personne de sa femme aussi une partie de son propre moi pour se livrer entièrement aux affaires publiques. 
$C^{\prime}$ est en fait la société toute entière qui s'héroïse. Pour répondre aux exigences d'une société en guerre et de la Terreur, la sensibilité se durcit, les sentiments s'aguerrissent. Les héros féminins et masculins sont l'expression vivante de ce déplacement sensible vers l'austérité spartiate, de cette collectivisation de tous les domaines de vie et de leur infiltration par la chose militaire. Ainsi l'apparition sur scène de l'amazone est le signe d'une mobilisation générale qui touche aussi les deux autres groupes de «faibles»: les vieillards et les enfants. La forte présence des femmes sur scène dans la pièce de la citoyenne Villiers suit cette même logique de guerre qui en fait disparaître les hommes.

\section{LA FEMME-VIRILE MODÈLE D'UNE ÉMANCIPATION FÉMININE?}

Les modèles d'héroïsme féminin sur scène offrent aux femmes une certaine liberté d'action pour remettre en question l'ordre défini des sexes et exprimer leurs aspirations et revendications. C'est l'amazone qui illustre le mieux cette tendance à l'émancipation. Mais la mère spartiate dans son rôle de citoyenne, prend elle aussi conscience de sa propre valeur, ce qui ébranle l'image traditionnelle des relations entre homme et femme. En même temps, cette liberté d'action est liée à un certain contexte social problématique. L'émancipation de la femme n'apparaît ici que dans la mesure où la société entière le permet et de plus, elle est dépendante des buts que se sont fixés les différentes fractions politiques. La virago, telle qu'elle apparaît dans la tragédie anonyme de 1795, Charlotte Corday, est foncièrement girondine. Quant à l'Epicharis de Légouvé, elle est une critique sous-jacente de la période de la Terreur: après Thermidor, on jouera la pièce comme illustration d'anti-robespierrisme.

Et si, dans les pièces pro-révolutionnaires, on laisse à la femme un champ d'action plus important, $c^{\prime}$ est bien parce que la situation politique en général et la politique du jour l'exigent, et non pas pour revendiquer une égalité civique et sociale de la femme. La citoyenne Villiers ellemême fait de la femme un instrument de la société patriarcale. Seule Olympe de Gouges utilise l'image de l'amazone à des fins proprement féministes. Mais déjà au moment de la première de la pièce, et pas seulement lors de son exécution, Olympe de Gouges se trouve en marge de la société: en marge à la fois des hommes et des femmes.

\section{ANNETTE GRACZYK}

Université de Marbourg, Allemagne

(traduit de l'allemand par Emmanuelle Deramond) 
Notes

$1 C^{\prime}$ est la version légèrement raccourcie et traduite en français d'une communication allemande, que j'ai présentée au colloque de Paderborn «Frauen - Literatur - Revolution», 19 - 22 sept. 1989, sous le titre de: «Der Zwang zum Heldentum: Das literarische Profil der Geschlechter im Theater der Französischen Revolution"; elle paraîtra avec les actes du colloque qui actuellement sont sous presse aux éditions Centaurus à Pfaffenweiler.

2 Cf. mon article "Débordement et ordre: Le Théâtre de la Révolution, média de masses entre 1789 et 1794", dans Dix-huitième Siècle (no 21, 1989, pp. 395-409); voir en outre mon essai d'introduction à mon anthologie commentée du théâtre révolutionnaire: Vorhang auf für die Revolution: Das französische Theater 1789-1795 (pièces traduites en allemand par T.S. Wohlfeil, A. Graczyk et H. Bartuschek, Weinheim/Berlin: Quadriga, 1989), ainsi que mon étude d'un auteur dramatique révolutionnaire: «Vergangenheit, Gegenwart und Zukunft: Der Dramatiker Louis-Benoît Picard während der Revolution» (dans Lendemains, dossier «La Littérature de la Révolution française", éd. A. Graczyk/ H. Hofer, no 55/56, 1989, pp. 57-70). - Pour une vue d'ensemble de la création littéraire sous la Révolution on se référa à l'ouvrage collectif Literatur der Französischen Revolution: Eine Einführung, éd. H. Krauß, Stuttgart: Metzler, 1988.

3 Cette typologie se base sur une étude plus globale de l'image de la femme dans le théâtre révolutionnaire, que j'examine ici tout particulièrement dans le contexte de la Terreur; cf. mon article: «'Die Frau ist doch kein Nichts in der Schöpfung' Frauenbild und Weiblichkeitssymbolik im Theater der Französischen Revolution,» dans Sklavin oder Bürgerin? Französische Revolution und neue Weiblichkeit 1760-1830, catalogue d'exposition du Musée historique de la ville de Francfort, éd. par Viktoria Schmidt-Linsenhoff, Marburg: Jonas, 1989, pp. 103-13.

4 Tragédie en cinq actes et en vers, par Légouvé, Citoyen français, Paris, an II; représentée pour la première fois au Théâtre de la République le 3 févr. 1794. Accessible sur microfiches dans Théâtre de la Révolution et de l'Empire, éd. M. Régaldo, Paris: Microédition Hachette, [1975].

5 Tragédie en trois actes et en vers, s.l. 1793; attribuée à Joseph-François Gamon par Jean-Alexis Rivoire dans Le Patriotisme dans le théâtre sérieux de la Révolution, 1789-1799, Paris, 1950. La pièce se trouve à la Bibliothèque Nationale parisienne sous la cote: Yth. 22 628. - Sur l'attentat de Charlotte Corday et son adaptation littéraire, cf. La Mort de Marat, ouvrage collectif sous la direction de Jean-Claude Bonnet, Paris, Flammarion, 1986, et dont l'article de Michel Delon se refère directement à 'La Fiction immédiate.'

6 Agricol Viala ou Le jeune héros de la Durance, fait historique et patriotique, par Philipon de la Madelaine, Paris, an II, représenté pour la première fois au Théâtre des Amis de la patrie, le 1er juillet 1794; citation p. 15. (Accessible sur microfiches dans Théâtre de la Révolution et de l'Empire, éd. M. Régaldo, ouvr. cité.) - «[...] la Liberté enfanta des héros, je veux que mon sexe en compte aussi", affirme Catherine dans L'Héroïne de Mithier, fait historique, en un acte et en prose, mêlé de vaudevilles, par les CC. Vée et Barral, Paris, an II, représenté pour la première fois au Théâtre Vaudeville, 20 août 1794.

7 La Mort du jeune Barra, ou Une Journée de la Vendée, drame historique en un acte, par le Citoyen Briois, Paris, an II; représenté pour la première fois au Théâtre de la République le 4 mai 1794.

8 Pièce en cinq actes et en prose, Paris, 1793, représentée pour la première fois au Théâtre de la République le 23 janvier 1793. Sur la femme-auteur cf. la biographie 
d'Olivier Blanc: Olympe de Gouges, Paris: Syros, 1981. - Sur l'engagement politique des femmes et la question de l'émancipation féminine sous la Révolution je renvoie à ma contribution bibliographique «Frauenrecht und Politik: weibliches Engagement in der Französischen Revolution, 1789-1795", dans: Das Achtzehnte Jahrhundert, Mitteilungen der Deutschen Gesellschaft für die Erforschung des 18. Jahrhunderts, Heft 2, 13/ 1989, pp. 111-25.

9 Tragédie en trois actes, par Marie-Joseph Chénier, Député à la Convention Nationale, Paris, 1793; représentée pour la première fois au Théâtre de la République le 9 février 1792.

10 Drame historique, en trois actes et en prose, par la citoyenne femme Villiers, Dijon, an II; première représentation au Théâtre de Dijon, le 25 mars 1794. La pièce est contenue dans Théâtre républicain. Répertoire du Théâtre Républicain ou Recueil de pièces imprimées avant, pendant et après la République Française (Réimpression de la collection de Lunel [Hérault], 1773-1822, 15 vol., Genève: Slatkine, 1986; tome VII, pièce 21). 